\title{
Ermakov-Painlevé II Symmetry Reduction of a Korteweg Capillarity System
}

Colin ROGERS ${ }^{\dagger}$ and Peter A. CLARKSON ${ }^{\ddagger}$

$\dagger$ Australian Research Council Centre of Excellence for Mathematics \& Statistics of Complex Systems, School of Mathematics, The University of New South Wales, Sydney, NSW2052, Australia

E-mail: c.rogers@unsw.edu.au

$\ddagger$ School of Mathematics, Statistics \& Actuarial Science, University of Kent, Canterbury, CT2 $7 F S$, UK

E-mail: P.A.Clarkson@kent.ac.uk

Received January 13, 2017, in final form March 15, 2017; Published online March 22, 2017 https://doi.org/10.3842/SIGMA.2017.018

\begin{abstract}
A class of nonlinear Schrödinger equations involving a triad of power law terms together with a de Broglie-Bohm potential is shown to admit symmetry reduction to a hybrid Ermakov-Painlevé II equation which is linked, in turn, to the integrable Painlevé XXXIV equation. A nonlinear Schrödinger encapsulation of a Korteweg-type capillary system is thereby used in the isolation of such a Ermakov-Painlevé II reduction valid for a multiparameter class of free energy functions. Iterated application of a Bäcklund transformation then allows the construction of novel classes of exact solutions of the nonlinear capillarity system in terms of Yablonskii-Vorob'ev polynomials or classical Airy functions. A Painlevé XXXIV equation is derived for the density in the capillarity system and seen to correspond to the symmetry reduction of its Bernoulli integral of motion.
\end{abstract}

Key words: Ermakov-Painlevé II equation; Painlevé capillarity; Korteweg-type capillary system; Bäcklund transformation

2010 Mathematics Subject Classification: 37J15; 37K10; 76B45; 76D45

\section{Introduction}

Giannini and Joseph [39], in a nonlinear optics context, introduced a class of symmetry reductions for a cubic nonlinear Schrödinger (NLS) equation

$$
i \Psi_{t}+\Psi_{x x}+\nu|\Psi|^{2} \Psi=0,
$$

with subscripts denoted partial derivatives, which resulted in the Painlevé II equation but with zero parameter $\alpha$

$$
\frac{d^{2} q}{d z^{2}}=2 q^{3}+z q
$$

see also [6, 49, 61]; the reduction of the NLS equation (1.1) to equation (1.2) was derived in $[37,86]$. Numerical integration led to the isolation of interesting non-stationary solutions both bounded and stable in shape for a restricted range of ratio of nonlinearily to dispersion. However, the absence of the Painlevé parameter $\alpha$ in that reduction does not allow the iterative construction of sequences of exact solutions via the Bäcklund transformation for the canonical Painlevé II $\left(\mathrm{P}_{\mathrm{II}}\right)$

$$
\frac{d^{2} q}{d z^{2}}=2 q^{3}+z q+\alpha
$$


with $\alpha$ a parameter, as given by Gambier [38] and Lukashevich [59]. Here, symmetry reduction to $\mathrm{P}_{\mathrm{II}}$ (1.3) or to a hybrid Ermakov-Painlevé II equation linked to the integrable Painlevé XXXIV (PXXXIV) equation

$$
\frac{d^{2} p}{d z^{2}}=\frac{1}{2 p}\left(\frac{d p}{d z}\right)^{2}+2 p^{2}-z p-\frac{\left(\alpha+\frac{1}{2}\right)^{2}}{2 p}
$$

with $\alpha$ a parameter, is obtained for a wide class of NLS equations which incorporates a triad of power law terms together with a de Broglie-Bohm potential term. It is remarked that NLS equations involving such triple power law nonlinearities arise in nonlinear optics (see [31, 91] and literature cited therein). Moreover, NLS equations containing a de Broglie-Bohm term also arise in the analysis of the propagation of optical beams [75, 90] as well as in cold plasma physics [56]. Under appropriate conditions, such 'resonant' NLS equations admit novel fusion or fission solitonic behaviour [55, 56, 67, 68].

The Ermakov-Painlevé II symmetry reduction is applied here to an NLS encapsulation of a nonlinear capillarity system with origin in classical work of Korteweg [54]. Iterated application of a Bäcklund transformation admitted by $\mathrm{P}_{\mathrm{II}}$ (1.3) permits the construction via the linked $\mathrm{P}_{\text {XXXIV }}$ equation of novel multi-parameter wave packet solutions to the capillarity system in terms of either Yablonskii-Vorob'ev polynomials or classical Airy functions. These are shown to be valid for a multi-parameter class of model specific free energy relations. An invariance of the $(1+1)$-dimensional Korteweg capillarity system under a one-parameter class of reciprocal transformations as recently set down in [80] allows the extension of the reduction procedure to a yet wider class of capillarity systems.

\section{A Ermakov-Painlevé II symmetry reduction}

Here, a class of $(1+1)$-dimensional nonlinear Schrödinger equations of the type

$$
i \Psi_{t}+\Psi_{x x}-\left[(1-\mathcal{C}) \frac{|\Psi|_{x x}}{|\Psi|}-i c \frac{|\Psi|_{x}}{|\Psi|^{2}}+\lambda|\Psi|^{2}+\mu|\Psi|^{2 m}+\nu|\Psi|^{2 n}\right] \Psi=0,
$$

which incorporates a de Broglie-Bohm potential $|\Psi|_{x x} /|\Psi|$ and a triad of power law terms is investigated under a symmetry reduction. Thus, constraints on the parameters in (2.1) are sought for which the class of NLS equations admits symmetry reduction either to the $\mathrm{P}_{\mathrm{II}}(1.3)$, with non-zero parameter $\alpha$, or to a hybrid Ermakov-Painlevé II equation under a wave packet ansatz

$$
\Psi=[\phi(\xi)+i \psi(\xi)] \exp (i \eta)
$$

with

$$
\xi=\alpha t+\beta t^{2}+\gamma x, \quad \eta=\gamma t^{3}+\delta t^{2}+\varepsilon \gamma t x+\zeta t+\lambda x .
$$

In the nonlinear optics context of [39] such a similarity transformation was used to reduce a standard cubic NLS equation to Painlevé II but with zero parameter $\alpha$ and resort was made to a numerical treatment. Asymptotic properties of $\mathrm{P}_{\mathrm{II}}(1.2)$ with $\alpha=0$ have been discussed by various authors, see, e.g., [2, 8, 23, 28, 34, 41, 62].

In the present case, on introduction of the wave packet ansatz (2.2) into (2.1), it is seen that

$$
\begin{aligned}
& \gamma^{2} \frac{d^{2} \phi}{d \xi^{2}}-\frac{d \psi}{d \xi}\left[2\left(\beta+\varepsilon \gamma^{2}\right) t+\alpha+2 \lambda \gamma\right]+\frac{c \gamma \psi}{|\Psi|^{3}}\left(\phi \frac{d \phi}{d \xi}+\psi \frac{d \psi}{d \xi}\right)-\Delta \phi=0 \\
& \gamma^{2} \frac{d^{2} \psi}{d \xi^{2}}+\frac{d \phi}{d \xi}\left[2\left(\beta+\varepsilon \gamma^{2}\right) t+\alpha+2 \lambda \gamma\right]-\frac{c \gamma \phi}{|\Psi|^{3}}\left(\phi \frac{d \phi}{d \xi}+\psi \frac{d \psi}{d \xi}\right)-\Delta \psi=0
\end{aligned}
$$


where

$$
\begin{aligned}
\Delta= & 3 \gamma t^{2}+2 \delta t+\varepsilon \gamma x+\zeta+(\varepsilon \gamma t+\lambda)^{2}+\lambda|\Psi|^{2}+\mu|\Psi|^{2 m}+\nu|\Psi|^{2 n} \\
& +\frac{s \gamma^{2}}{|\Psi|^{4}}\left\{\left[\phi \frac{d^{2} \phi}{d \xi^{2}}+\psi \frac{d^{2} \psi}{d \xi^{2}}+\left(\frac{d \phi}{d \xi}\right)^{2}+\left(\frac{d \psi}{d \xi}\right)^{2}\right]\left(\phi^{2}+\psi^{2}\right)-\left(\phi \frac{d \phi}{d \xi}+\psi \frac{d \psi}{d \xi}\right)^{2}\right\},
\end{aligned}
$$

with $s=1-\mathcal{C}$. The relations $(2.3)$ together show that

$$
\begin{aligned}
& \gamma^{2}\left(\frac{d^{2} \phi}{d \xi^{2}} \psi-\frac{d^{2} \psi}{d \xi^{2}} \phi\right)-\left(\phi \frac{d \phi}{d \xi}+\psi \frac{d \psi}{d \xi}\right)\left[2\left(\beta+\varepsilon \gamma^{2}\right) t+\alpha+2 \lambda \gamma\right] \\
& +\frac{c \gamma}{|\Psi|}\left(\phi \frac{d \phi}{d \xi}+\psi \frac{d \psi}{d \xi}\right)=0
\end{aligned}
$$

whence it is required that

$$
\beta+\varepsilon \gamma^{2}=0,
$$

in which case equation (2.5) admits the integral

$$
\gamma^{2}\left(\frac{d \phi}{d \xi} \psi-\frac{d \psi}{d \xi} \phi\right)-\frac{1}{2}(\alpha+2 \lambda \gamma)|\Psi|^{2}+c \gamma|\Psi|=\mathcal{I},
$$

where $\mathcal{I}$ is an arbitrary constant of motion.

On use of the relation

$$
\left[\phi \frac{d^{2} \phi}{d \xi^{2}}+\psi \frac{d^{2} \psi}{d \xi^{2}}+\left(\frac{d \phi}{d \xi}\right)^{2}+\left(\frac{d \psi}{d \xi}\right)^{2}\right]\left(\phi^{2}+\psi^{2}\right)-\left(\phi \frac{d \phi}{d \xi}+\psi \frac{d \psi}{d \xi}\right)^{2}=|\Psi|^{3} \frac{d^{2}|\Psi|}{d \xi^{2}}
$$

it is seen that (2.4) yields, if $\beta \neq 0$,

$$
\begin{aligned}
\Delta= & \gamma \beta^{-1}\left(\varepsilon^{2} \gamma+3\right)(\xi-\alpha t-\gamma x)+2(\delta+\varepsilon \gamma \lambda) t+\varepsilon \gamma x+\zeta+\lambda^{2} \\
& +\lambda|\Psi|^{2}+\mu|\Psi|^{2 m}+\nu|\Psi|^{2 n}+\frac{s \gamma^{2}}{|\Psi|} \frac{d^{2}|\Psi|}{d \xi^{2}} \\
= & \varepsilon \xi+\zeta+\lambda^{2}+\lambda|\Psi|^{2}+\mu|\Psi|^{2 m}+\nu|\Psi|^{2 n}+\frac{s \gamma^{2}}{|\Psi|} \frac{d^{2}|\Psi|}{d \xi^{2}},
\end{aligned}
$$

on setting

$$
\beta \varepsilon=\gamma\left(3+\varepsilon^{2} \gamma\right), \quad \alpha \varepsilon=2(\delta+\varepsilon \gamma \lambda) .
$$

Moreover, equations (2.3) again combine to show that

$$
\gamma^{2}\left(\phi \frac{d^{2} \phi}{d \xi^{2}}+\psi \frac{d^{2} \psi}{d \xi^{2}}\right)+\left(\frac{d \phi}{d \xi} \psi-\frac{d \psi}{d \xi} \phi\right)(\alpha+2 \lambda \gamma)-\Delta|\Psi|^{2}=0
$$

whence, on use of the identity

$$
\left(\phi^{2}+\psi^{2}\right)\left[\left(\frac{d \phi}{d \xi}\right)^{2}+\left(\frac{d \psi}{d \xi}\right)^{2}\right]-\left(\frac{d \phi}{d \xi} \psi-\frac{d \psi}{d \xi} \phi\right)^{2} \equiv\left(\phi \frac{d \phi}{d \xi}+\psi \frac{d \psi}{d \xi}\right)^{2},
$$

together with (2.7), it is seen that

$$
\gamma^{2}\left[|\Psi|^{3} \frac{d^{2}|\Psi|}{d \xi^{2}}-\left(\frac{d \phi}{d \xi} \psi-\frac{d \psi}{d \xi} \phi\right)^{2}\right]+(\alpha+2 \lambda \gamma)\left(\frac{d \phi}{d \xi} \psi-\frac{d \psi}{d \xi} \phi\right)|\Psi|^{2}-\Delta|\Psi|^{4}=0 .
$$


The latter, by virtue of the integral of motion (2.6) and the expression (2.8) for $\Delta$ now produces a nonlinear equation in the amplitude $|\Psi|$, namely

$$
\begin{aligned}
\frac{d^{2}|\Psi|}{d \xi^{2}} & +\left[c_{1}+c_{2} \xi\right]|\Psi|+c_{3}|\Psi|^{3}+c_{4}|\Psi|^{2 m+1}+c_{5}|\Psi|^{2 n+1}+\frac{c_{6}}{|\Psi|}+\frac{c_{7}}{|\Psi|^{2}} \\
& =\frac{\mathcal{I}^{2}}{(1-s) \gamma^{4}|\Psi|^{3}},
\end{aligned}
$$

where the constants $c_{1}, c_{2}, \ldots, c_{7}$ are given by

$$
\begin{aligned}
& c_{1}=\frac{(\alpha-\delta / \varepsilon)^{2}-\gamma^{2}\left(\zeta+\lambda^{2}\right)}{(1-s) \gamma^{4}}, \quad c_{2}=\frac{\varepsilon}{(s-1) \gamma^{2}}, \quad c_{3}=\frac{\lambda}{(s-1) \gamma^{2}}, \\
& c_{4}=\frac{\mu}{(s-1) \gamma^{2}}, \quad c_{5}=\frac{\nu}{(s-1) \gamma^{2}}, \quad c_{6}=\frac{c^{2}}{(s-1) \gamma^{2}}, \quad c_{7}=\frac{2 c \mathcal{I}}{(1-s) \gamma^{3}},
\end{aligned}
$$

and it is required that $s \neq 1$.

Below a triad of cases is set down in which the amplitude equation (2.10) reduces either directly to $\mathrm{P}_{\mathrm{II}}$ or to a hybrid Ermakov-Painlevé II equation subsequently shown to be integrable.

Case (i) $\mathcal{I}=0 ; m=-\frac{1}{2} ; n=-1$.

In this case with

$$
c_{1}=0, \quad c_{2}=-1, \quad c_{3}=-2, \quad c_{4}=-\alpha, \quad c_{5}+c_{6}=0,
$$

the amplitude equation reduces directly to the Painlevé II equation

$$
\frac{d^{2}|\Psi|}{d \xi^{2}}=2|\Psi|^{3}+\xi|\Psi|+\alpha
$$

corresponding to the symmetry reduction via the ansatz (2.2) of the class of NLS equations

$$
i \Psi_{t}+\Psi_{x x}-\left[(1-\mathcal{C}) \frac{|\Psi|_{x x}}{|\Psi|}-i c \frac{|\Psi|_{x}}{|\Psi|^{2}}+\mathcal{C} \gamma^{2}|\Psi|^{2}+\frac{\mathcal{C} \gamma^{2} \alpha}{|\Psi|}-\frac{c^{2}}{|\Psi|^{2}}\right] \Psi=0
$$

Case (ii) $\mathcal{I}=0 ; c=0 ; m=-\frac{1}{2} ; n=0$.

Here, with

$$
c_{1}=-c_{5}, \quad c_{2}=-1, \quad c_{3}=-2, \quad c_{4}=-\alpha,
$$

the $\mathrm{P}_{\mathrm{II}}$ equation (2.12) again results, while the associated class of NLS equations (2.1) becomes

$$
i \Psi_{t}+\Psi_{x x}-\left[(1-\mathcal{C}) \frac{|\Psi|_{x x}}{|\Psi|}+\mathcal{C} \gamma^{2}|\Psi|^{2}+\frac{\mathcal{C} \gamma^{2} \alpha}{|\Psi|}+\nu\right] \Psi=0
$$

It is remarked that in the absence of the de Broglie-Bohm term, a time-independent NLS equation of this type (2.13) incorporating a nonlinearity $\sim|\Psi|^{-1}$ has been derived 'ab initio' in [84] via a geometric model which describes stationary states of supercoiled DNA. 
Case (iii) $\mathcal{I} \neq 0 ; c=0 ; m=-\frac{1}{2} ; n=0$.

In this case, equation (2.10) reduces to a hybrid 'Ermakov-Painlevé II' equation of the type

$$
\frac{d^{2}|\Psi|}{d \xi^{2}}+\varepsilon|\Psi|^{3}+(\delta \xi+\zeta)|\Psi|=\frac{\sigma}{|\Psi|^{3}},
$$

and which will be subsequently seen to be linked to $\mathrm{P}_{\mathrm{XXXIV}}$ (1.4). It is recalled that the classical Ermakov equation with roots in [30], namely

$$
\frac{d^{2} \mathcal{E}}{d \xi^{2}}+\omega(\xi) \mathcal{E}=\frac{\sigma}{\mathcal{E}^{3}}
$$

admits a nonlinear superposition principle readily derived via a Lie group approach as in [76, 81].

In the subsequent application to the Korteweg capillarity system it will be the ErmakovPainlevé II symmetry reduction that will be exploited. With a positive solution $|\Psi|$ of $(2.14)$ to hand, the corresponding class of exact solutions for $\Psi$ in the wave packet representation (2.2) is obtained via the integral of motion (2.6). Thus, the latter yields

$$
\gamma^{2} \frac{d}{d \xi}\left[\tan ^{-1}\left(\frac{\phi}{\psi}\right)\right]-\alpha+\frac{\delta}{\varepsilon}+\frac{c \gamma}{|\Psi|}=\frac{\mathcal{I}}{|\Psi|^{2}}
$$

whence, on integration

$$
\gamma^{2} \tan ^{-1}\left(\frac{\phi}{\psi}\right)=\left(\alpha-\frac{\delta}{\varepsilon}\right) \xi-c \gamma \int \frac{1}{|\Psi|} d \xi+\mathcal{I} \int \frac{1}{|\Psi|^{2}} d \xi
$$

where use has been made of the relation (2.9). Accordingly, with $V=\phi / \psi$, it is seen that $\phi, \psi$ in the original wave packet representation are given by the relations

$$
\phi= \pm \frac{|\Psi| V}{\sqrt{1+V^{2}}}, \quad \psi= \pm \frac{|\Psi|}{\sqrt{1+V^{2}}}
$$

In the sequel, the link between the Ermakov-Painlevé II equation (2.14) and PXxxIV (1.4) is used to construct novel classes of wave packet solutions of a nonlinear Korteweg capillarity system in terms of Yablonskii-Vorob'ev polynomials or classical Airy functions via the iterated application of the Bäcklund transformation for $\mathrm{P}_{\mathrm{II}}$ due to Gambier [38] and Lukashevich [59].

\section{The capillarity system}

In [4], Antanovskii derived the isothermal capillarity system with continuity equation

$$
\rho_{t}+\operatorname{div}(\rho \mathbf{v})=0,
$$

augmented by the momentum equation

$$
\mathbf{v}_{t}+\mathbf{v} \bullet \nabla \mathbf{v}+\nabla\left[\frac{\delta(\rho \mathcal{E})}{\delta \rho}-\Pi\right]=\mathbf{0}
$$

where $\rho$ is the density, $\mathbf{v}$ velocity and $\mathcal{E}\left(\rho,|\nabla \rho|^{2} / \rho\right)$ is the specific free energy. Herein, the standard variational derivative notation

$$
\frac{\delta \Theta}{\delta \rho}=\frac{\partial \Theta}{\partial \rho}-\nabla \cdot\left[\frac{\partial \Theta}{\partial \mathcal{A}} \nabla \rho\right],
$$


is adopted with $\mathcal{A}=\frac{1}{2}|\nabla \rho|^{2}$. In the above $\Pi$ is an external potential, commonly taken to be that due to gravity, in which case $\Pi=-\rho g$. The classical Korteweg capillarity system as set down in [54] is retrieved in the specialisation

$$
\mathcal{E}(\mathcal{A}, \rho)=\frac{\kappa(\rho) \mathcal{A}}{\rho}, \quad \kappa(\rho)>0,
$$

in which case, the momentum equation becomes

$$
\mathbf{v}_{t}+\mathbf{v} \bullet \nabla \mathbf{v}-\nabla\left[\kappa(\rho) \nabla^{2} \rho+\frac{1}{2}|\nabla \rho|^{2} \frac{d \kappa(\rho)}{d \rho}+\Pi\right]=0 .
$$

The classical Boussinesq capillarity system, in turn, is retrieved as the specialisation with $\kappa$ constant in this Korteweg system. A system analogous to the Boussinesq model arises 'mutatis mutandis' in plasma physics [9].

In the case of irrotationality with $\mathbf{v}=\nabla \Phi$, the momentum equation (3.1b) admits the Bernoulli integral

$$
\Phi_{t}+\frac{1}{2}|\nabla \Phi|^{2}+\frac{\delta}{\delta \rho}(\rho \mathcal{E})-\Pi=\mathcal{B}(t)
$$

and on introduction of the Madelung transformation [60]

$$
\Psi=\rho^{1 / 2} \exp \left(\frac{1}{2} i \Phi\right)
$$

the capillarity system (3.1) may be encapsulated in the generalised NLS-type equation

$$
i \Psi_{t}+\nabla^{2} \Psi+\left[-\frac{\nabla^{2}|\Psi|}{|\Psi|}-\frac{1}{2} \frac{\delta(\rho \mathcal{E})}{\delta \rho}+\frac{1}{2} \Pi\right] \Psi=0,
$$

incorporating a de Broglie-Bohm potential term.

It was observed by Antanovskii et al. in [5] that if $\Pi=0$ and

$$
\mathcal{E}\left(\frac{1}{2}|\nabla \rho|^{2}, \rho\right)=\mathcal{C} \frac{|\nabla \rho|^{2}}{2 \rho^{2}}+\nu \rho+\frac{\tau}{\rho}
$$

then (3.3) reduces, if $\mathcal{C}=1$, to the cubic nonlinear Schrödinger equation

$$
i \Psi_{t}+\nabla^{2} \Psi-\nu|\Psi|^{2} \Psi=0 .
$$

If, on the other hand, $\mathcal{C} \neq 1$, it is seen that reduction is obtained to a 'resonant' NLS-type equation [78]

$$
i \Psi_{t}+\nabla^{2} \Psi+\left[(\mathcal{C}-1) \frac{\nabla^{2}|\Psi|}{|\Psi|}-\nu|\Psi|^{2}\right] \Psi=0
$$

Moreover, if $\mathcal{C}>0$ as in the present capillarity context, (3.5) may be transformed to a standard cubic NLS equation with the de Broglie-Bohm term removed (see, e.g., [73]). Thus, in $1+1$ dimensions with three-parameter model energy $\mathcal{E}\left(\frac{1}{2}|\nabla \rho|^{2}, \rho\right)$ of the type (3.4) reduction is made to a canonical integrable NLS equation. The capillarity system encapsulated in the $(1+1)$ dimensional version of (3.5) then becomes amenable to established methods of soliton theory such as inverse scattering procedures and inherits admittance of invariance under a Bäcklund transformation together with concomitant nonlinear superposition principle (see, e.g., [1, 77, 79] and literature cited therein). It is noted that a gravitational potential term $\Pi=-\rho g$ is readily 
accommodated in the above reduction. Detailed qualitative properties of capillarity systems with model laws of the type (3.4) with Kármán-Tsien-type law

$$
\kappa(\rho)=\mathcal{C} / \rho, \quad \mathcal{C}>0
$$

have been recently set down in [16] while travelling wave propagation in $(1+1)$-dimensional capillarity theory has been investigated in [10].

Here, a more general class of model energy $\mathcal{E}\left(\frac{1}{2}|\nabla \rho|^{2}, \rho\right)$ laws is considered, namely that with

$$
\mathcal{E}=\frac{\kappa(\rho)|\nabla \rho|^{2}}{2 \rho}+\frac{\mathcal{R}(\rho)}{\rho}
$$

so that, with $\Pi=0,(3.3)$ produces the class of NLS equations

$$
i \Psi_{t}+\nabla^{2} \Psi-\left[\left(1+\frac{d \kappa}{d \rho}|\Psi|^{4}\right) \frac{\nabla^{2}|\Psi|}{|\Psi|}-\frac{1}{2}\left(\kappa(\rho)+\frac{d \kappa}{d \rho}|\Psi|^{2}\right)(\nabla|\Psi|)^{2}+\frac{1}{2} \frac{d \mathcal{R}}{d \rho}\right] \Psi=0 .
$$

Thus, capillarity systems encapsulated in (3.7) are isolated which may be aligned with NLS equations of the type (2.1) in the case $c=0, m=-2, n=0$. This occurs for the multiparameter class of model energy laws with

$$
\mathcal{E}\left(\frac{1}{2}|\nabla \rho|^{2}, \rho\right)=\mathcal{C} \frac{|\nabla \rho|^{2}}{2 \rho^{2}}+\lambda \rho-\frac{2 \mu}{\rho^{2}}+2 \nu+\frac{\tau}{\rho},
$$

where $\lambda, \mu, \nu$ and $\tau$ together with $\mathcal{C}>0$ are real constants. Importantly, this includes in the case $\mu=0$ the class which has been recently subject to a detailed qualitative analysis in [16]. The $\kappa(\rho)$ capillarity relation is seen to be of the Kármán-Tsien type (3.6). Here,

$$
\lambda=-\mathcal{C} \gamma^{2} c_{3}, \quad \mu=-\mathcal{C} \gamma^{2} c_{4}, \quad \nu=-\mathcal{C} \gamma^{2} c_{5},
$$

in accordance with the relations (2.11). The associated class of NLS equations

$$
i \Psi_{t}+\Psi_{x x}-\left[(\mathcal{C}-1) \frac{|\Psi|_{x x}}{|\Psi|}+\lambda|\Psi|^{2}+\frac{\mu}{|\Psi|^{4}}+\nu\right] \Psi=0
$$

hence, admits symmetry reduction via the wave packet ansatz (2.2) to the hybrid ErmakovPainlevé II equation (cf. (2.10))

$$
\frac{d^{2}|\Psi|}{d \xi^{2}}+\left[c_{1}+c_{5}+c_{2} \xi\right]|\Psi|+c_{3}|\Psi|^{3}=\frac{\sigma}{|\Psi|^{3}}
$$

where

$$
\sigma=\frac{1}{\mathcal{C} \gamma^{2}}\left[\left(\frac{\mathcal{I}}{\gamma}\right)^{2}+\mu\right] .
$$

Interestingly, this symmetry reduction to an integrable Ermakov-Painlevé II equation will be admitted by Korteweg-type capillarity systems with the particular model energy laws of the type discussed in [16].

Under the translation $\zeta=\xi+\left(c_{1}+c_{5}\right) / c_{2}$, with $c_{2} \neq 0$, (3.10) becomes

$$
\frac{d^{2}|\Psi|}{d \zeta^{2}}+c_{2} \zeta|\Psi|+c_{3}|\Psi|^{3}=\frac{\sigma}{|\Psi|^{3}}
$$


where the Madelung relation (3.2) shows that, in the present capillarity context $|\Psi|=\rho^{1 / 2}$. Thus, $\rho^{1 / 2}$ is governed by a hybrid Ermakov-Painlevé II equation while in terms of the density $\rho$ it is seen that (3.12) produces

$$
\frac{d^{2} \rho}{d \zeta^{2}}=\frac{1}{2 \rho}\left(\frac{d \rho}{d \zeta}\right)^{2}-2 c_{3} \rho^{2}-2 c_{2} \zeta \rho+\frac{2 \sigma}{\rho},
$$

which is equivalent to $\mathrm{P}_{\mathrm{XXXIV}}$ (1.4) (through a rescaling of the variables). This link between the Ermakov-Painlevé II equation (3.12) and PXxxIV (1.4) has been noted previously in the context of a Painlevé reduction of a classical Nernst-Planck electrodiffusion system in [3]. We remark that the special case of equation (3.13) with $c_{3}=0$ was considered by Gambier [38, pp. 27-28], who linearised the equation. Multiplying (3.13) with $c_{3}=0$ by $\rho$ and differentiating gives

$$
\frac{d^{3} \rho}{d \zeta^{3}}=4 \zeta \frac{d \rho}{d \zeta}+2 \rho
$$

which has solution

$$
\rho(\zeta)=C_{1} \operatorname{Ai}^{2}(z)+C_{2} \operatorname{Ai}(z) \operatorname{Bi}(z)+C_{3} \operatorname{Bi}^{2}(z), \quad z=-c_{2}^{1 / 3} \zeta,
$$

with $C_{1}, C_{2}$ and $C_{3}$ constants. The solution $\rho(\zeta)$ given by (3.14) satisfies (3.13) only if $c_{3}=0$, $\sigma=0$ and $4 C_{1} C_{2}=C_{3}^{2}$.

In the sequel, it is convenient to proceed with

$$
c_{2}=-\frac{1}{2}, \quad c_{3}=-1, \quad \sigma=-\frac{1}{4}\left(\alpha+\frac{1}{2}\right)^{2},
$$

whence

$$
\varepsilon=\frac{1}{2} \mathcal{C} \gamma^{2}>0, \quad \lambda=\mathcal{C} \gamma^{2}>0, \quad\left(\alpha+\frac{1}{2}\right)^{2}=-\frac{4}{\lambda}\left[\left(\frac{\mathcal{I}}{\gamma}\right)^{2}+\mu\right],
$$

where the latter requires that $\mu<-\mathcal{C I}^{2} / \lambda<0$. The Ermakov-Painlevé II equation (3.12) is then linked to $\mathrm{P}_{\text {XXXIV }}(1.4)$ via the relation $\rho=|\Psi|^{2}>0$.

The well-known connection, in turn, between $\mathrm{P}_{\mathrm{II}}$ (1.3) and $\mathrm{P}_{\mathrm{XXXIV}}$ (1.4) is readily derived via the Hamiltonian system

$$
\frac{d q}{d z}=\frac{\partial \mathcal{H}_{\mathrm{II}}}{\partial p}, \quad \frac{d p}{d z}=-\frac{\partial \mathcal{H}_{\mathrm{II}}}{\partial q},
$$

where the Hamiltonian $\mathcal{H}_{\mathrm{II}}(p, q, z ; \alpha)$ is given by

$$
\mathcal{H}_{\mathrm{II}}(p, q, z ; \alpha)=\frac{1}{2} p^{2}-\left(q^{2}+\frac{1}{2} z\right) p-\left(\alpha+\frac{1}{2}\right) q,
$$

leading to the coupled pair of nonlinear equations

$$
\frac{d q}{d z}=p-q^{2}-\frac{1}{2} z, \quad \frac{d p}{d z}=2 q p+\alpha+\frac{1}{2},
$$

(see $[44,66]$ ). Elimination of $p$ and $q$ successively in (3.16) duly leads to the $\mathrm{P}_{\mathrm{II}}(1.3)$ and $\mathrm{P}_{\mathrm{XXXIV}}$ (1.4). Thus, in the present capillarity context, the density distribution $\rho(\zeta)$ is given by

$$
\rho(\zeta)=\frac{d w}{d \zeta}+w^{2}+\frac{1}{2} \zeta
$$


where $w(\zeta)$ is governed by the $\mathrm{P}_{\mathrm{II}}$ equation

$$
\frac{d^{2} w}{d \zeta^{2}}=2 w^{3}+\zeta w+\alpha
$$

Here, the concern is necessarily restricted to solutions of $\mathrm{P}_{\mathrm{XXXIV}}(1.4)$ in regions in which $\rho$ is positive. Interestingly, the importance of positive solutions of $\mathrm{P}_{\mathrm{XXXIV}}$ (1.4) also arises naturally in the setting of two-ion electro-diffusion. Thus, in the electrolytic context of $[7,13]$, the scaled electric field $Y$ was shown to be governed by the $\mathrm{P}_{\text {II }}$ equation

$$
\frac{d^{2} Y}{d z^{2}}=2 Y^{3}+z Y+\alpha
$$

and associated ion concentrations by

$$
p_{ \pm}= \pm \frac{d Y}{d z}+Y^{2}+\frac{1}{2} z
$$

with parameter

$$
\alpha=\frac{1-A_{-} / A_{+}}{2\left(1+A_{-} / A_{+}\right)},
$$

and $A_{ \pm}=-\Phi_{ \pm} / D_{ \pm}, \Phi_{ \pm}$being the fluxes of the ion concentrations and $D_{ \pm}$diffusivity constants arising in the Einstein relation. Thus, it is seen that the ion concentrations, which are necessarily positive, are governed by $\mathrm{P}_{\text {XXXIV }}$ (1.4). This positivity constraint was examined in detail in [7] for exact solutions in terms of either Yablonskii-Vorob'ev polynomials or classical Airy functions as induced by the iterated action of the Bäcklund transformation of [59] for $\mathrm{P}_{\text {II }}$ (1.3). The results apply 'mutatis mutandis' in the present capillarity context.

\section{Iterated action of a Bäcklund transformation}

Here, the consequences of the following well-known Bäcklund transformation for $\mathrm{P}_{\mathrm{II}}$ (1.3) are applied in the present capillarity context.

Theorem 4.1. If $q_{\alpha}(z)=q(z ; \alpha)$ is a solution of $\mathrm{P}_{\mathrm{II}}$ (1.3) with parameter $\alpha$, then

$$
\begin{aligned}
& q_{\alpha+1}(z)=-q_{\alpha}(z)-\frac{2 \alpha+1}{2 q_{\alpha}^{\prime}(z)+2 q_{\alpha}^{2}(z)+z}, \\
& q_{\alpha-1}(z)=-q_{\alpha}(z)-\frac{2 \alpha-1}{2 q_{\alpha}^{\prime}(z)-2 q_{\alpha}^{2}(z)+z},
\end{aligned}
$$

are solution of $\mathrm{P}_{\mathrm{II}}$ with respective parameters $\alpha+1$ and $\alpha-1$.

Proof. See Gambier [38] and Lukashevich [59].

The iteration of the Bäcklund transformations (4.1) allows the generation of all known exact solutions of $\mathrm{P}_{\mathrm{II}}(1.3)$.

We note that eliminating $q_{\alpha}^{\prime}(z)$ in (4.1) yields the nonlinear difference equation

$$
\frac{\alpha+\frac{1}{2}}{q_{\alpha+1}+q_{\alpha}}+\frac{\alpha-\frac{1}{2}}{q_{\alpha}+q_{\alpha-1}}+2 q_{\alpha}^{2}+z=0,
$$

which is known as an alternative form of discrete Painlevé I [33]. 
Theorem 4.2. If $q_{\alpha}=q(z ; \alpha)$ and $p_{\alpha}=p(z ; \alpha)$ are solutions of $\mathrm{P}_{\mathrm{II}}(1.3)$ and $\mathrm{P}_{\mathrm{XXXIV}}$ with parameter $\alpha$ respectively, then

$$
\begin{aligned}
& q_{\alpha+1}=-q_{\alpha}-\frac{2 \alpha+1}{2 p_{\alpha}}, \\
& q_{\alpha-1}=-q_{\alpha}+\frac{2 \alpha-1}{2 p_{\alpha}-4 q_{\alpha}^{2}+2 z}, \\
& p_{\alpha+1}=-p_{\alpha}+\left(q_{\alpha}+\frac{2 \alpha+1}{2 p_{\alpha}}\right)^{2}+z, \\
& p_{\alpha-1}=-p_{\alpha}+2 q_{\alpha}^{2}+z .
\end{aligned}
$$

Proof. See Okamoto [66]; also [35].

\subsection{Rational solutions}

The iterative action of the above Bäcklund transformation on the seed solution $q=0$ of $\mathrm{P}_{\mathrm{II}}$ with $\alpha=0$ produces the subsequent sequence of rational solutions

$$
q_{n}(z)=\frac{d}{d z} \ln \frac{Q_{n-1}(z)}{Q_{n}(z)}, \quad n \in \mathbb{N}
$$

corresponding to the Painlevé parameters $\alpha=n$, for $n \in \mathbb{N}$, where the $Q_{n}(z)$ are the YablonskiiVorob'ev polynomials determined by the quadratic recurrence relations

$$
Q_{n+1} Q_{n-1}=z Q_{n}^{2}+4\left\{\left(\frac{d Q_{n}}{d z}\right)^{2}-Q_{n} \frac{d^{2} Q_{n}}{d z^{2}}\right\},
$$

with $Q_{-1}(z)=Q_{0}(z)=1[89,92]$; see also $[18,19,22,48,50,87]$. The $Q_{n}(z)$ are monic polynomials of degree $\frac{1}{2} n(n+1)$ with each term possessing the same degree modulo 3 . Moreover, on use of the invariance under

$$
q(z, \alpha) \rightarrow-q(z ;-\alpha),
$$

it is seen that $\mathrm{P}_{\mathrm{II}}(1.3)$ also admits the associated class of rational solutions

$$
q_{-n}(z)=\frac{d}{d z} \ln \frac{Q_{n}(z)}{Q_{n-1}(z)}, \quad n \in \mathbb{N}
$$

corresponding to the Painlevé parameters $\alpha=-n$, for $n \in \mathbb{N}$. The rational solutions of $\mathrm{P}_{\mathrm{XXXIV}}$ (1.4) are given by

$$
p_{n}(z)=\frac{1}{2} z-2 \frac{d^{2}}{d z^{2}} \ln Q_{n}(z) \equiv \frac{Q_{n+1}(z) Q_{n-1}(z)}{2 Q_{n}^{2}(z)}, \quad n \in \mathbb{N},
$$

corresponding to the parameters $\alpha=n$, with $n \in \mathbb{N}$.

It is clear from the recurrence relation (4.3) that the $Q_{n}(z)$ are rational functions, though it is not obvious that they are polynomials since one is dividing by $Q_{n-1}(z)$ at every iteration. In fact it is somewhat remarkable that the $Q_{n}(z)$ are polynomials. Taneda [87], used an algebraic method to prove that the functions $Q_{n}(z)$ defined by (4.3) are indeed polynomials, see also [36]. The Yablonskii-Vorob'ev polynomials $Q_{n}(z)$ can also be expressed as determinants, see [22, 46, 47]. Clarkson and Mansfield [22] investigated the locations of the roots of the Yablonskii-Vorob'ev polynomials in the complex plane and showed that these roots have a very regular, approximately triangular structure; the term "approximate" is used since the patterns 
are not exact triangles as the roots lie on arcs rather than straight lines. Recently Bertola and Bothner [11] and Buckingham and Miller [14, 15] have studied the Yablonskii-Vorob'ev polynomials $Q_{n}(z)$ in the limit as $n \rightarrow \infty$ and shown that the roots lie in a "triangular region" with elliptic sides which meet with interior angle $\frac{2}{5} \pi$, suggesting a limit to a solution of Painlevé I $\left(\mathrm{P}_{\mathrm{I}}\right)$. Indeed Buckingham and Miller [15] show that in the limit as $n \rightarrow \infty$, the rational solution $q_{n}(z)$ of $\mathrm{P}_{\mathrm{II}}$ tends to the tritronquée solution of $\mathrm{P}_{\mathrm{I}}$ due to Boutroux [12], which no poles (of large modulus) except in one sector of angle $\frac{2}{5} \pi$ (see also [45]).

In the sequel, attention is restricted to the case with invariants $\mathcal{I}=\mathcal{I}_{n} \neq 0$ so that similarity reduction of the capillarity system via (2.2) leads to consideration of a Ermakov-Painlevé II equation in the amplitude $|\Psi|$ and, in turn, density $\rho$ as determined by $P_{X X X I V}$ (3.13). Thus, the density distribution $\rho=\rho_{+}$associated with the class of exact solutions (4.2) of $\mathrm{P}_{\mathrm{II}}(1.3)$ in terms of Yablonskii-Vorob'ev polynomials with $z=\zeta$ may be shown to be given by rational expressions derived via (3.12) to adopt the form (see [18, 19, 22])

$$
\rho_{+}(\zeta ; n)=\frac{Q_{n+1}(\zeta) Q_{n-1}(\zeta)}{2 Q_{n}^{2}(\zeta)}, \quad \rho_{+}(\zeta ; 0)=\frac{1}{2} \zeta
$$

as subsequently employed in the two-ion electolytic boundary value problems investigated in [7]. Therein, the positivity of members of the class of rational solutions (4.5) of PXxxIV on appropriate regions has been delimited. In the present capillarity context, in such regions where $\rho=\rho_{+}$ is positive, the class of solutions (4.5) is associated with wave packet representations (2.2) with

$$
\begin{aligned}
& \phi(\xi)= \pm \frac{V(\xi)}{\sqrt{1+V^{2}(\xi)}} \sqrt{\frac{Q_{n+1}(\xi) Q_{n-1}(\xi)}{2 Q_{n}^{2}(\xi)}}, \\
& \psi(\xi)= \pm \frac{1}{\sqrt{1+V^{2}(\xi)}} \sqrt{\frac{Q_{n+1}(\xi) Q_{n-1}(\xi)}{2 Q_{n}^{2}(\xi)}}
\end{aligned}
$$

where $V(\xi)$ is given by, in view of $(2.15)$,

$$
\begin{aligned}
\gamma^{2} \tan ^{-1} V(\xi) & =\left(\alpha-\frac{\delta}{\varepsilon}\right) \xi+2 \mathcal{I}_{n} \int^{\xi} \frac{Q_{n}^{2}(s)}{Q_{n+1}(s) Q_{n-1}(s)} d s \\
& =\left(\alpha-\frac{\delta}{\varepsilon}\right) \xi+\frac{2 \mathcal{I}_{n}}{2 n+1} \ln \frac{Q_{n+1}(\xi)}{Q_{n-1}(\xi)},
\end{aligned}
$$

since

$$
\int^{\xi} \frac{Q_{n}^{2}(s)}{Q_{n+1}(s) Q_{n-1}(s)} d s=\frac{1}{2 n+1} \ln \frac{Q_{n+1}(\xi)}{Q_{n-1}(\xi)} .
$$

This result (4.8) follows since the Yablonskii-Vorob'ev polynomials $Q_{n}(\xi)$ satisfy the bilinear relation

$$
\frac{d Q_{n+1}}{d \xi} Q_{n-1}-\frac{d Q_{n-1}}{d \xi} Q_{n+1}=(2 n+1) Q_{n}^{2}
$$

which is proved in $[36,87]$ (see also [50]). Likewise, there are associated classes of wave packet representations corresponding to the rational solution $q_{-n}(z)$ given by (4.4) which are determined by the relations (4.6), (4.7) but with the transposition $n \leftrightarrow n-1$.

\subsection{Airy-type solutions}

The iterated action of the Bäcklund transformations (4.1) may, in addition, be used to generate exact solutions of $\mathrm{P}_{\mathrm{II}}(1.3)$ with parameters $\alpha= \pm \frac{1}{2}, \pm \frac{3}{2}, \ldots$ in terms of classical Airy 
functions [21]. Thus, in particular, if $\alpha=\frac{1}{2}$ then $\mathrm{P}_{\mathrm{II}}$ (1.3) admits the exact solution

$$
q\left(z ; \frac{1}{2}\right)=-\frac{d}{d z} \ln \varphi(z)
$$

where $\varphi(z)$ is governed by the classical Airy function

$$
\frac{d^{2} \varphi}{d z^{2}}+\frac{1}{2} z \varphi=0
$$

Iteration of the Bäcklund transformation (4.1a) with the Airy-type seed solution (4.9) generates as infinite sequence of exact solutions

$$
q\left(z ; n-\frac{1}{2}\right)=\frac{d}{d z} \ln \frac{u_{n-1}(z)}{u_{n}(z)}, \quad n \in \mathbb{N},
$$

where the sequence $\left\{u_{\ell}(z)\right\}$, for $\ell \geq 0$, is determined by the recurrence relation (Toda equation)

$$
u_{n+1} u_{n-1}=4\left\{\left(\frac{d u_{n}}{d z}\right)^{2}-u_{n} \frac{d^{2} u_{n}}{d z^{2}}\right\},
$$

with initial values

$$
u_{0}(z)=1, \quad u_{1}(z)=\varphi(z) .
$$

The $u_{n}(z)$, for $n \geq 2$, are homogeneous polynomials of degree $n$ in $\varphi(z)$ and $\varphi^{\prime}(z)$, i.e., have the form

$$
u_{n}(z)=\sum_{j=0}^{n} a_{n, j} \varphi^{j}\left(\frac{d \varphi}{d z}\right)^{n-j},
$$

where $a_{n, j}(z)$ are polynomials in $z$ and $\varphi(z)$ is the solution of (4.10) given by

$$
\varphi(z)=\cos (\vartheta) \operatorname{Ai}\left(-2^{-1 / 3} z\right)+\sin (\vartheta) \operatorname{Bi}\left(-2^{-1 / 3} z\right),
$$

with $\operatorname{Ai}(x)$ and $\operatorname{Bi}(x)$ the Airy functions and $\vartheta$ an arbitrary constant.

The analogous Airy-type solutions of PxxxIV (1.4) are given by

$$
p\left(z ; n-\frac{1}{2}\right)=-2 \frac{d^{2}}{d z^{2}} \ln u_{n} \equiv \frac{u_{n-1} u_{n+1}}{2 u_{n}^{2}}, \quad n \in \mathbb{N},
$$

for the parameter $\alpha=n-\frac{1}{2}$.

The Airy-type solutions of $\mathrm{P}_{\mathrm{II}}$ (1.3) and $\mathrm{P}_{\mathrm{XXXIV}}$ (1.4) can also be expressed in terms of determinants, as described in the following theorem.

Theorem 4.3. Let $\tau_{n}(z)$ be the Hankel $n \times n$ determinant

$$
\tau_{n}(z)=\left[\frac{d^{j+k}}{d z^{j+k}} \varphi(z)\right]_{j, k=0}^{n-1}, \quad n \geq 1,
$$

with $\varphi(z)$ given by (4.13) and $\tau_{0}(z)=1$, then for $n \geq 1$,

$$
q\left(z ; n-\frac{1}{2}\right)=\frac{d}{d z} \ln \frac{\tau_{n-1}(z)}{\tau_{n}(z)}, \quad p\left(z ; n-\frac{1}{2}\right)=-2 \frac{d^{2}}{d z^{2}} \ln \tau_{n}(z),
$$

respectively satisfy $\mathrm{P}_{\mathrm{II}}$ (1.3) and $\mathrm{P}_{\mathrm{XXXIV}}(1.4)$ with $\alpha=n-\frac{1}{2}$. 
Proof. See Flaschka and Newell [32], Okamoto [66]; also [21, 35].

We remark that recently it was shown that Airy-type solutions of $\mathrm{P}_{\mathrm{II}}$ (1.3) and $\mathrm{P}_{\mathrm{XXXIV}}$ (1.4) which depend only on the Airy function $\operatorname{Ai}(x)$ have a completely different structure to those which involve a linear combination of the Airy functions $\operatorname{Ai}(x)$ and $\operatorname{Bi}(x)$, see [21]. In particular, for $n \in 2 \mathbb{N}$ the solution (4.14) of $\mathrm{P}_{\text {XXXIV }}$ (1.4) has no poles on the real axis when $\varphi(z)=$ $\operatorname{Ai}\left(-2^{-1 / 3} z\right)$ and decays algebraically as $z \rightarrow \pm \infty$. This special solution arose in a study of the double scaling limit of unitary random matrix ensembles by Its, Kuijlaars, and Östensson $[42,43]$, who identify the solution as a tronquée solution of $\mathrm{P}_{\mathrm{XXXIV}}$, i.e., has no poles in a sector of the complex plane (see also [21]).

The iterative application of the Bäcklund transformations (4.1) to generate Airy-type solutions of $\mathrm{P}_{\mathrm{II}}$ (1.3) has been used to solve boundary value problems associated with the classical Nernst-Planck system for two-ion electro-diffusion [7, 74]. The repeated action of the Bäcklund transformations in this electrolytic setting has been recently associated with quantised fluxes of ionic species in [13]. It is remarked that the Painlevé structure underlying a multi-ion electrodiffusion model has been systematically investigated in [26].

In the present capillarity context, the density distributions associated with the class of exact solutions of $\mathrm{P}_{\mathrm{II}}$ (1.3) determined by the relations (4.11), (4.12) are given by [7]

$$
\rho\left(\xi ; n-\frac{1}{2}\right)=\frac{u_{n-1}(\xi) u_{n+1}(\xi)}{2 u_{n}^{2}(\xi)}, \quad \rho\left(\xi ;-\frac{1}{2}\right)=0,
$$

where $u_{n}(z)$ is given by (4.12). The physical requirement of positivity of these solutions corresponding to the specialisation $\varphi(z)=\operatorname{Ai}\left(-2^{-1 / 3} z\right)$ in (4.13), i.e., when $\vartheta=0$, has been examined in detail in the electrolytic context of [7]. The implications of the results carry over to the present capillarity study.

In general, with regard to the velocity magnitude $v=|\mathbf{v}|$, alignment of the Madelung transformation (3.2) with (2.2) produces the velocity potential relation

$$
\Phi=2 \tan ^{-1}\left(\frac{\psi+\phi \tan \eta}{\phi-\psi \tan \eta}\right)=2\left[\tan ^{-1}\left(\frac{\psi}{\phi}\right)+\eta\right]
$$

on use of the identity

$$
\tan ^{-1}\left(\frac{x+y}{1-x y}\right) \equiv \tan ^{-1} x+\tan ^{-1} y .
$$

Integration of the invariant relation (2.6) where in the present context $c=0$, yields

$$
-\gamma^{2} \tan ^{-1}\left(\frac{\psi}{\phi}\right)=\left(\alpha-\frac{\delta}{\varepsilon}\right) \xi+2 \mathcal{I} \int^{\xi} \frac{u_{n}^{2}(s)}{u_{n+1}(s) u_{n-1}(s)} d s=\left(\alpha-\frac{\delta}{\varepsilon}\right) \xi+\frac{\mathcal{I}}{n} \ln \frac{u_{n+1}(\xi)}{u_{n-1}(\xi)},
$$

since

$$
\int^{\xi} \frac{u_{n}^{2}(s)}{u_{n+1}(s) u_{n-1}(s)} d s=\frac{1}{2 n} \ln \frac{u_{n+1}(\xi)}{u_{n-1}(\xi)} .
$$

The result (4.16) holds as the $u_{n}(\xi)$ satisfy the bilinear relation

$$
\frac{d u_{n+1}}{d \xi} u_{n-1}-\frac{d u_{n-1}}{d \xi} u_{n+1}=2 n u_{n}^{2}
$$

which follows from Theorem 4.2. Consequently (4.15) yields

$$
|\mathbf{v}|=-\frac{2}{\gamma^{2}}\left[\gamma \frac{\partial}{\partial \xi}+(\varepsilon \gamma t+\lambda) \frac{\partial}{\partial \eta}\right]\left[\left(\alpha-\frac{\delta}{\varepsilon}\right) \xi+\mathcal{I} \int \frac{1}{|\Psi|^{2}} d \xi+\eta\right]
$$


whence,

$$
v=-\frac{2 \mathcal{I}}{\gamma \rho(\xi)}+2 \varepsilon \gamma t-\frac{\alpha}{\gamma},
$$

on use of the relation (2.9). Insertion of the latter expression into the momentum equation of the capillarity system, namely

$$
v_{t}+v v_{x}+\left[\frac{\delta}{\delta \rho}(\rho \mathcal{E})\right]_{x}=0
$$

on integration, produces the Bernoulli integral

$$
\frac{2 \mathcal{I}^{2}}{\gamma^{2} \rho^{2}}+2 \varepsilon \gamma x+\frac{\delta}{\delta \rho}(\rho \mathcal{E})=\mathcal{B}(t)
$$

Here (3.8) shows that

$$
\rho \mathcal{E}=\mathcal{C} \frac{\gamma^{2}}{2 \rho} \rho_{x}^{2}+\lambda \rho^{2}+\frac{2 \mu}{\rho}+2 \nu \rho+\tau,
$$

whence,

$$
\frac{\delta}{\delta \rho}(\rho \mathcal{E})=\frac{\partial}{\partial \rho}(\rho \mathcal{E})-\mathcal{C} \frac{\partial}{\partial x}\left(\frac{\rho_{x}}{\rho}\right)=2 \lambda \rho+\frac{2 \mu}{\rho^{2}}+2 \nu+\mathcal{C}\left(\frac{\rho_{x}^{2}}{2 \rho^{2}}-\frac{\rho_{x x}}{\rho}\right),
$$

in which $\rho(x, t)=R(\xi)$ with $\xi=\alpha t+\beta t^{2}+\gamma x$ so that $\rho_{x}=\gamma R^{\prime}(\xi)$. Thus,

$$
\frac{2 \mathcal{I}^{2}}{\gamma^{2} R^{2}}+2 \varepsilon \gamma x+2 \lambda R+\frac{2 \mu}{R^{2}}+2 \nu+\mathcal{C} \gamma^{2}\left[\frac{1}{2 R^{2}}\left(\frac{d R}{d \xi}\right)^{2}-\frac{1}{R} \frac{d^{2} R}{d \xi^{2}}\right]=\mathcal{B}(t),
$$

and with $\mathcal{B}(t)=-2 \varepsilon\left(\alpha t+\beta t^{2}\right)$, an equation equivalent to $\operatorname{PXXXIV}(1.4)$ for the density $\rho(\zeta)$ is retrieved, namely

$$
\frac{d^{2} \rho}{d \zeta^{2}}=\frac{1}{2 \rho}\left(\frac{d \rho}{d \zeta}\right)^{2}+\frac{2 \lambda \rho^{2}}{\mathcal{C} \gamma^{2}}+\frac{2 \varepsilon \zeta \rho}{\mathcal{C} \gamma^{2}}+\frac{2\left(\mathcal{I}^{2}+\gamma^{2} \mu\right)}{\mathcal{C} \gamma^{4} \rho}, \quad \zeta=\xi+\frac{c_{1}+c_{5}}{c_{2}}
$$

which aligns with (3.13) in view of the relations (3.9), (3.11) and (3.15). Thus, it is seen that, remarkably, in the present context $\mathrm{P}_{\mathrm{XXXIV}}$ is associated with the density $\rho$ corresponding to a symmetry reduction of the Bernoulli integral of motion of the capillarity system.

\section{Invariance under a reciprocal transformation}

The application of reciprocal-type transformations to $(1+1)$-dimensional nonlinear physical systems has its origin in the isolation of novel invariance properties in gasdynamics and magnetogasdynamics [70, 71]. They have been subsequently applied to both obtain analytic solution to moving boundary problems of Stefan-type [72] and to link integrable systems of modern soliton theory (see, e.g., [27, 65] together with [79] and work cited therein). In $3+1$ dimensions, reciprocal-type transformations have been shown to have application in discontinuity wave propagation theory [29].

In the present capillarity context, the $(1+1)$-dimensional version of the system (3.1) with $\Pi=0$, namely

$$
\rho_{t}+(\rho v)_{x}=0, \quad v_{t}+v v_{x}+\left[\frac{\delta}{\delta \rho}(\rho \mathcal{E})\right]_{x}=0,
$$


was recently shown in [80] to be invariant under the one-parameter $(\chi)$ class of reciprocal-type transformations

$$
\begin{aligned}
& \rho^{*}=\frac{\rho}{1+\chi \rho}, \quad q^{*}=q, \quad \mathcal{E}^{*}\left(\mathcal{A}^{*}, \rho^{*}\right)=\mathcal{E}(\mathcal{A}, \rho), \quad \mathcal{A}^{*}=\frac{\mathcal{A}}{(1+\chi \rho)^{6}}, \\
& d x^{*}=(1+\chi \rho) d x-\chi \rho q d t, \quad d t^{*}=d t, \quad 0<|1+\chi \rho|<\infty,
\end{aligned}
$$

where $\mathcal{A}=\frac{1}{2} \rho_{x}^{2}$. A direct corollary of this result is that the $(1+1)$-dimensional Korteweg-type capillarity system

$$
\begin{aligned}
& \rho_{t}+(\rho v)_{x}=0 \\
& v_{t}+v v_{x}+\left(-\kappa(\rho) \rho_{x x}-\frac{1}{2} \frac{d \kappa}{d \rho} \rho_{x}^{2}+\frac{d \mathcal{R}}{d \rho}\right)_{x}=0,
\end{aligned}
$$

is invariant under the one-parameter class of reciprocal transformations

$$
d x^{*}=(1+\chi \rho) d x-\chi \rho q d t, \quad d t^{*}=d t, \quad \rho^{*}=\frac{\rho}{1+\chi \rho}, \quad q^{*}=q,
$$

augmented by the relations

$$
\kappa^{*}=(1+\chi \rho)^{5} \kappa, \quad \mathcal{R}^{*}=\frac{\mathcal{R}}{1+\chi \rho} .
$$

This invariant transformation may be applied to seed solutions of the capillarity system (5.1) as previously determined in terms of Yablonskii-Vorob'ev polynomials or classical Airy functions to construct extended $\chi$-dependent classes of exact solutions valid for model energy laws $\mathcal{E}^{*}$ with $\chi$-deformed Kármán-Tsien capillarity relation

$$
\kappa^{*}=\frac{\mathcal{C}}{\rho^{*}\left(1-\chi \rho^{*}\right)^{4}},
$$

together with

$$
\mathcal{R}^{*}=\frac{\lambda \rho^{* 2}}{1-\chi \rho^{*}}-2 \mu \frac{\left(1-\chi \rho^{*}\right)^{2}}{\rho^{*}}+2 \nu \rho^{*}+\tau\left(1-\chi \rho^{*}\right) .
$$

The original $\kappa(\rho), \mathcal{R}(\rho)$ associated with the $\mathrm{P}_{\text {XXXIV }}$ reduction are retrieved in the limit $\chi \rightarrow 0$.

\section{General perspectives on model laws in continuum mechanics and solitonic connections: conclusion}

Here, model energy laws have been isolated for which a Korteweg-type capillarity system admits symmetry reduction to a integrable hybrid Ermakov-Painlevé equation. A Bäcklund and reciprocal transformation have been used in turn to generate novel classes of exact solutions and to extend the range of the reduction. The derivation of multi-parameter model constitutive laws for which systems in nonlinear continuum mechanics become analytically tractable via the application of Bäcklund or reciprocal transformations has an extensive literature. Thus, in gasdynamics, Loewner [57, 58] applied matrix Bäcklund transformations to construct model constitutive laws for which the classical hodograph equations may be systematically reduced to appropriate tractable canonical forms in subsonic, transonic and supersonic flow régimes. The celebrated Kármán-Tsien two-parameter pressure-density model law of [88] as extensively applied in subsonic gasdynamics, arises as a particular reduction. The Bäcklund transformations as introduced in the model gas law context of [58], suitably interpreted and extended, 
remarkably, turn out to have application in $(2+1)$-dimensional soliton theory $[52,53]$. In nonlinear elastodynamics, model multi-parameter stress-strain laws were constructed in [17] which allow the analytic treatment of aspects of shock-less pulse propagation in bounded nonlinear elastic media. Comparison of experimental stress-strain relations with such model laws was investigated, in particular, for the dynamic compression of saturated soil, dry sand and clay silt. A Bäcklund transformation may be introduced at the level of the stress-strain laws for the uniaxial Lagrangian elastodynamic system treated in [17]. The single action of this Bäcklund transformation to the classical Hooke's law generates the multi-parameter class of $(T, e)$ laws applied extensively therein. Moreover application of a nonlinear superposition principle associated with the Bäcklund transformation permits the construction of more general model nonlinear $(T, e)$-laws for which the $(1+1)$-dimensional elastodynamic system may be iteratively reduced to that associated with the canonical Hooke's law. There is again a remarkable solitonic connection in that the nonlinear superposition principle acting on the $(T, e)$-laws turns out to be nothing but the permutability theorem for the potential Korteweg-de Vries hierarchy (see, e.g., [79]).

In nonlinear elastostatics, model stress-deformation laws have been introduced by Neuber in $[63,64]$ in connection with the problem of determining the stress-distribution in shear-strained isotropic prismatical bodies. Loewner-type Bäcklund transformations were applied in [24] to the Neuber elastostatic system to solve a class of indentation boundary value problems for both Neuber-Sokolovsky and power law model stress-deformation relations. The application of model B-H and D-E constitutive laws in the analysis of the propagation of plane polarised electromagnetic waves through nonlinear dielectric media has been described in [51]. In general terms, it was shown in [82] that model constitutive laws as constructed via the Bäcklund approach introduced by Loewner, corresponds to solitonic solutions generated by a Darboux-type transformation.

With regard to reciprocal transformations and their role in the construction of model constitutive laws one may cite, in particular, the investigation of Storm in [85] concerning heat conduction in simple monatomic metals. Therein, a class of model $\left(c_{p}(T), k(T)\right)$ temperature $T$-dependent laws was introduced for which a $(1+1)$-dimensional nonlinear heat conduction equation may be reduced via a reciprocal transformation to the classical linear heat equation. The applicability of these model laws was justified in [85] for appropriate specific heat $c_{p}(T)$ and thermal conductivity $k(T)$ over wide temperature ranges for such materials as aluminium, silver, sodium, cadium, zinc, copper and lead. This kind of reduction via a reciprocal transformation may be extended to hyperbolic systems that correspond to multi-parameter model laws that arise in Cattaneo-type conduction and nonlinear visco-elasticity (see, e.g., [83]).

The preceding attests to the importance and wide range of physical applications of model constitutive laws in nonlinear continuum mechanics together with intriguing solitonic connections. The six classical Painlevé equations arise in a wide range of physical applications and play a fundamental role in modern soliton theory (see, e.g., [20, 25, 34, 40]). In the present work, model multi-parameter specific energy laws have been isolated which allow symmetry reduction of a Korteweg capillarity system to consideration of a hybrid Ermakov-Painlevé II equation and thereby to the linked integrable $\mathrm{P}_{\mathrm{XxxIV}}$ equation. In conclusion, it is remarked that the Ermakov-Painlevé II symmetry reduction presented here is also valid for a superfluidity model system involving a de Broglie Bohm potential as set down in [69].

\section{References}

[1] Ablowitz M.J., Clarkson P.A., Solitons, nonlinear evolution equations and inverse scattering, London Mathematical Society Lecture Note Series, Vol. 149, Cambridge University Press, Cambridge, 1991.

[2] Ablowitz M.J., Segur H., Solitons and the inverse scattering transform, SIAM Studies in Applied Mathematics, Vol. 4, Society for Industrial and Applied Mathematics (SIAM), Philadelphia, Pa., 1981. 
[3] Amster P., Rogers C., On a Ermakov-Painlevé II reduction in three-ion electrodiffusion. A Dirichlet boundary value problem, Discrete Contin. Dyn. Syst. 35 (2015), 3277-3292.

[4] Antanovskii L.K., Microscale theory of surface tension, Phys. Rev. E 54 (1996), 6285-6290.

[5] Antanovskii L.K., Rogers C., Schief W.K., A note on a capillarity model and the nonlinear Schrödinger equation, J. Phys. A: Math. Gen. 30 (1997), L555-L557.

[6] Assanto G., Minzoni A.A., Smyth N.F., On optical Airy beams in integrable and non-integrable systems, Wave Motion 52 (2015), 183-193.

[7] Bass L., Nimmo J.J.C., Rogers C., Schief W.K., Electrical structures of interfaces: a Painlevé II model, Proc. R. Soc. Lond. Ser. A Math. Phys. Eng. Sci. 466 (2010), 2117-2136.

[8] Bassom A.P., Clarkson P.A., Law C.K., McLeod J.B., Application of uniform asymptotics to the second Painlevé transcendent, Arch. Rational Mech. Anal. 143 (1998), 241-271, solv-int/9609005.

[9] Belashov V.Yu., Vladimirov S.V., Solitary waves in dispersive complex media. Theory, simulation, applications, Springer Series in Solid-State Sciences, Vol. 149, Springer-Verlag, Berlin, 2005.

[10] Benzoni-Gavage S., Planar traveling waves in capillary fluids, Differential Integral Equations 26 (2013), 439-485.

[11] Bertola M., Bothner T., Zeros of large degree Vorob'ev-Yablonski polynomials via a Hankel determinant identity, Int. Math. Res. Not. 2015 (2015), 9330-9399, arXiv:1401.1408.

[12] Boutroux P., Recherches sur les transcendantes de M. Painlevé et l'étude asymptotique des équations différentielles du second ordre, Ann. Sci. École Norm. Sup. (3) 30 (1913), 255-375.

[13] Bracken A.J., Bass L., Rogers C., Bäcklund flux quantization in a model of electrodiffusion based on Painlevé II, J. Phys. A: Math. Theor. 45 (2012), 105204, 20 pages, arXiv:1201.0673.

[14] Buckingham R.J., Miller P.D., Large-degree asymptotics of rational Painlevé-II functions: noncritical behaviour, Nonlinearity 27 (2014), 2489-2578, arXiv:1310.2276.

[15] Buckingham R.J., Miller P.D., Large-degree asymptotics of rational Painlevé-II functions: critical behaviour, Nonlinearity 28 (2015), 1539-1596, arXiv:1406.0826.

[16] Carles R., Danchin R., Saut J.-C., Madelung, Gross-Pitaevskii and Korteweg, Nonlinearity 25 (2012), 2843-2873, arXiv:1111.4670.

[17] Cekirge H.M., Varley E., Large amplitude waves in bounded media I. Reflexion and transmission of large amplitude shockless pulses at an interface, Philos. Trans. Roy. Soc. London Ser. A 273 (1973), 261-313.

[18] Clarkson P.A., Painlevé equations - nonlinear special functions, J. Comput. Appl. Math. 153 (2003), 127140.

[19] Clarkson P.A., Remarks on the Yablonskii-Vorob'ev polynomials, Phys. Lett. A 319 (2003), $137-144$.

[20] Clarkson P.A., Painlevé equations - nonlinear special functions, in Orthogonal polynomials and special functions, Lecture Notes in Math., Vol. 1883, Springer, Berlin, 2006, 331-411.

[21] Clarkson P.A., On Airy solutions of the second Painlevé equation, Stud. Appl. Math. 137 (2016), 93-109, arXiv:1510.08326.

[22] Clarkson P.A., Mansfield E.L., The second Painlevé equation, its hierarchy and associated special polynomials, Nonlinearity 16 (2003), R1-R26.

[23] Clarkson P.A., McLeod J.B., A connection formula for the second Painlevé transcendent, Arch. Rational Mech. Anal. 103 (1988), 97-138.

[24] Clements D.L., Rogers C., On the theory of stress concentration for shear-strained prismatical bodies with a non-linear stress-strain law, Mathematika 22 (1975), 34-42.

[25] Conte R. (Editor), The Painlevé property. One century later, CRM Series in Mathematical Physics, SpringerVerlag, New York, 1999.

[26] Conte R., Rogers C., Schief W.K., Painlevé structure of a multi-ion electrodiffusion system, J. Phys. A: Math. Theor. 40 (2007), F1031-F1040, arXiv:0711.0615.

[27] Degasperis A., Holm D.D., Hone A.N.W., A new integrable equation with peakon solutions, Theoret. and Math. Phys. 133 (2002), 1463-1474, nlin.SI/0205023.

[28] Deift P.A., Zhou X., Asymptotics for the Painlevé II equation, Comm. Pure Appl. Math. 48 (1995), $277-337$.

[29] Donato A., Ramgulam U., Rogers C., The 3+1-dimensional Monge-Ampère equation in discontinuity wave theory: application of a reciprocal transformation, Meccanica 27 (1992), 257-262. 
[30] Ermakov V.P., Second-order differential equations: conditions for complete integrability, Univ. Izv. Kiev 20 (1880), no. 9, 1-25, see Appl. Anal. Discrete Math. 2 (2008), 123-145.

[31] Eslami M., Mirzazadeh M., Optical solitons with Biswas-Milovic equation for power law and dual-power law nonlinearities, Nonlinear Dynam. 83 (2016), 731-738.

[32] Flaschka H., Newell A.C., Monodromy- and spectrum-preserving deformations. I, Comm. Math. Phys. 76 (1980), 65-116.

[33] Fokas A.S., Grammaticos B., Ramani A., From continuous to discrete Painlevé equations, J. Math. Anal. Appl. 180 (1993), 342-360.

[34] Fokas A.S., Its A.R., Kapaev A.A., Novokshenov V.Yu., Painlevé transcendents. The Riemann-Hilbert approach, Mathematical Surveys and Monographs, Vol. 128, Amer. Math. Soc., Providence, RI, 2006.

[35] Forrester P.J., Witte N.S., Application of the $\tau$-function theory of Painlevé equations to random matrices: PIV, PII and the GUE, Comm. Math. Phys. 219 (2001), 357-398, math-ph/0103025.

[36] Fukutani S., Okamoto K., Umemura H., Special polynomials and the Hirota bilinear relations of the second and the fourth Painlevé equations, Nagoya Math. J. 159 (2000), 179-200.

[37] Gagnon L., Winternitz P., Lie symmetries of a generalised nonlinear Schrödinger equation. II. Exact solutions, J. Phys. A: Math. Gen. 22 (1989), 469-497.

[38] Gambier B., Sur les équations différentielles du second ordre et du premier degré dont l'intégrale générale est a points critiques fixes, Acta Math. 33 (1910), 1-55.

[39] Giannini J.A., Joseph R.I., The role of the second Painlevé transcendent in nonlinear optics, Phys. Lett. A 141 (1989), 417-419.

[40] Gromak V.I., Laine I., Shimomura S., Painlevé differential equations in the complex plane, De Gruyter Studies in Mathematics, Vol. 28, Walter de Gruyter \& Co., Berlin, 2002.

[41] Hastings S.P., McLeod J.B., A boundary value problem associated with the second Painlevé transcendent and the Korteweg-de Vries equation, Arch. Rational Mech. Anal. 73 (1980), 31-51.

[42] Its A.R., Kuijlaars A.B.J., Östensson J., Critical edge behavior in unitary random matrix ensembles and the thirty-fourth Painlevé transcendent, Int. Math. Res. Not. 2008 (2008), no. 9, rnn017, 67 pages, arXiv:0704.1972.

[43] Its A.R., Kuijlaars A.B.J., Östensson J., Asymptotics for a special solution of the thirty fourth Painlevé equation, Nonlinearity 22 (2009), 1523-1558, arXiv:0811.3847.

[44] Jimbo M., Miwa T., Monodromy preserving deformation of linear ordinary differential equations with rational coefficients. II, Phys. D 2 (1981), 407-448.

[45] Joshi N., Kitaev A.V., On Boutroux's tritronquée solutions of the first Painlevé equation, Stud. Appl. Math. 107 (2001), 253-291.

[46] Kajiwara K., Masuda T., A generalization of determinant formulae for the solutions of Painlevé II and XXXIV equations, J. Phys. A: Math. Gen. 32 (1999), 3763-3778, solv-int/9903014.

[47] Kajiwara K., Ohta Y., Determinant structure of the rational solutions for the Painlevé II equation, J. Math. Phys. 37 (1996), 4693-4704, solv-int/9607002.

[48] Kametaka Y., Noda M., Fukui Y., Hirano S., A numerical approach to Toda equation and Painlevé II equation, Mem. Fac. Eng. Ehime Univ. 9 (1986), 1-24.

[49] Kaminer I., Segev M., Christodoulides D.N., Self-accelerating self-trapped optical beams, Phys. Rev. Lett. 106 (2011), 213903, 4 pages.

[50] Kaneko M., Ochiai H., On coefficients of Yablonskii-Vorob'ev polynomials, J. Math. Soc. Japan 55 (2003), 985-993, math.CA/0205178.

[51] Kazakia J.Y., Venkataraman R., Propagation of electromagnetic waves in a nonlinear dielectric slab, Z. Angew. Math. Phys. 26 (1975), 61-76.

[52] Konopelchenko B., Rogers C., On (2+1)-dimensional nonlinear systems of Loewner-type, Phys. Lett. A 158 (1991), 391-397.

[53] Konopelchenko B., Rogers C., On generalized Loewner systems: novel integrable equations in $2+1$ dimensions, J. Math. Phys. 34 (1993), 214-242.

[54] Korteweg D.K., Sur la forme que prennent les équations du mouvement des fluides si l'on tient compte des forces capillaires causées par des variations de densité considérables mais continues et sur la théorie de la capillarité dans l'hypothèse d'une variation continue de la densité, Arch. Néerl. 6 (1901), 1-24. 
[55] Lee J.-H., Pashaev O.K., Solitons of the resonant nonlinear Schrödinger equation with nontrivial boundary conditions: Hirota bilinear method, Theoret. and Math. Phys. 152 (2007), 991-1003, nlin.SI/0611003.

[56] Lee J.-H., Pashaev O.K., Rogers C., Schief W.K., The resonant nonlinear Schrödinger equation in cold plasma physics. Application of Bäcklund-Darboux transformations and superposition principles, J. Plasma Phys. 73 (2007), 257-272.

[57] Loewner C., A transformation theory of the partial differential equations of gas dynamics, Tech. Notes Nat. Adv. Comm. Aeronaut. 1950 (1950), 1-56.

[58] Loewner C., Generation of solutions of systems of partial differential equations by composition of infinitesimal Baecklund transformations, J. Anal. Math. 2 (1953), 219-242.

[59] Lukashevich N.A., On the theory of Painlevé's second equation, Differ. Equ. 7 (1971), 853-854.

[60] Madelung E., Quantentheorie in hydrodynamischen Form, Z. Phys. 40 (1926), 322-326.

[61] Mahalov A., Suslov S.K., An "Airy gun": self-accelerating solutions of the time-dependent Schrödinger equation in vacuum, Phys. Lett. A 377 (2012), 33-38.

[62] Miles J.W., On the second Painlevé transcendent, Proc. Roy. Soc. London Ser. A 361 (1978), $277-291$.

[63] Neuber H., Kerbspannungslehre. Grundlagen für genaue Festigkeitsberechnung mit Berücksichtigung von Konstruktionsform und Werkstoff, Texts and Monographs in Physics, Springer-Verlag, Berlin, 1958.

[64] Neuber H., Theory of stress concentration for shear-strained prismatical bodies with arbitrary nonlinear stress-strain law, J. Appl. Mech. 28 (1961), 544-550.

[65] Oevel W., Rogers C., Gauge transformations and reciprocal links in $2+1$ dimensions, Rev. Math. Phys. 5 (1993), 299-330.

[66] Okamoto K., Studies on the Painlevé equations. III. Second and fourth Painlevé equations, $P_{\mathrm{II}}$ and $P_{\mathrm{IV}}$, Math. Ann. 275 (1986), 221-255.

[67] Pashaev O.K., Lee J.-H., Resonance solitons as black holes in Madelung fluid, Modern Phys. Lett. A 17 (2002), 1601-1619, hep-th/9810139.

[68] Pashaev O.K., Lee J.-H., Rogers C., Soliton resonances in a generalized nonlinear Schrödinger equation, J. Phys. A: Math. Theor. 41 (2008), 452001, 9 pages.

[69] Roberts P.H., Berloff N.G., The nonlinear Schrödinger equation as a model of superfluidity, in Quantum Vortex Dynamics and Superfluid Turbulence, Lecture Notes in Phys., Vol. 571, Editors C.F. Barenghi, R.J. Donnelly, W.F. Vinen, Springer, Berlin, 2001, 235-257.

[70] Rogers C., Reciprocal relations in non-steady one-dimensional gasdynamics, Z. Angew. Math. Phys. 19 (1968), 58-63.

[71] Rogers C., Invariant transformations in non-steady gasdynamics and magnetogasdynamics, Z. Angew. Math. Phys. 20 (1969), 370-382.

[72] Rogers C., On a class of moving boundary problems in nonlinear heat conduction: application of a Bäcklund transformation, Internat. J. Non-Linear Mech. 21 (1986), 249-256.

[73] Rogers C., Integrable substructure in a Korteweg capillarity model. A Kármán-Tsien type constitutive relation, J. Nonlinear Math. Phys. 21 (2014), 74-88.

[74] Rogers C., Bassom A.P., Schief W.K., On a Painlevé II model in steady electrolysis: application of a Bäcklund transformation, J. Math. Anal. Appl. 240 (1999), 367-381.

[75] Rogers C., Malomed B., Chow K., An H., Ermakov-Ray-Reid systems in nonlinear optics, J. Phys. A: Math. Theor. 43 (2010), 455214, 15 pages.

[76] Rogers C., Ramgulam U., A nonlinear superposition principle and Lie group invariance: application in rotating shallow water theory, Internat. J. Non-Linear Mech. 24 (1989), 229-236.

[77] Rogers C., Schief W.K., Intrinsic geometry of the NLS equation and its auto-Bäcklund transformation, Stud. Appl. Math. 101 (1998), 267-287.

[78] Rogers C., Schief W.K., Geodesic motion in multidimensional unified gauge theories, Nuovo Cimento B 114 (1999), 1409-1412.

[79] Rogers C., Schief W.K., Bäcklund and Darboux transformations. Geometry and modern applications in soliton theory, Cambridge Texts in Applied Mathematics, Cambridge University Press, Cambridge, 2002.

[80] Rogers C., Schief W.K., The classical Korteweg capillarity system: geometry and invariant transformations, J. Phys. A: Math. Theor. 47 (2014), 345201, 20 pages. 
[81] Rogers C., Schief W.K., Winternitz P., Lie-theoretical generalization and discretization of the Pinney equation, J. Math. Anal. Appl. 216 (1997), 246-264.

[82] Schief W.K., Rogers C., Loewner transformations: adjoint and binary Darboux connections, Stud. Appl. Math. 100 (1998), 391-422.

[83] Seymour B., Varley E., A Bäcklund transformation for a nonlinear telegraph equation, in Wave Phenomena: Modern Theory and Applications, North-Holland Mathematics Studies, Vol. 97, Editors C. Rogers and T.M. Moodie, North-Holland, Amsterdam, 1984, 299-306.

[84] Shi Y., Hearst J.E., The Kirchoff elastic rod, the nonlinear Schrödinger equation, and DNA supercoiling, J. Chem. Phys. 101 (1994), 5186-5200.

[85] Storm M.L., Heat conduction in simple metals, J. Appl. Phys. 22 (1951), 940-951.

[86] Tajiri M., Similarity reductions of the one- and two-dimensional nonlinear Schrödinger equations, J. Phys. Soc. Japan 52 (1983), 1908-1917.

[87] Taneda M., Remarks on the Yablonskii-Vorob'ev polynomials, Nagoya Math. J. 159 (2000), 87-111.

[88] Tsien H.-S., Two-dimensional subsonic flow of compressible fluids, J. Aeronaut. Sci. 6 (1939), 399-407.

[89] Vorob'ev A.P., On the rational solutions of the second Painlevé equation, Differ. Equ. 1 (1965), 79-81.

[90] Wagner W.G., Haus H.A., Marburger J.H., Large-scale self-trapping of optical beams in the paraxial ray approximation, Phys. Rev. 175 (1968), 256-266.

[91] Xu Y., Suarez P., Milovic D., Khan K.R., Mahmood M.F., Biswas A., Belic M., Raman solitons in nanoscale optical waveguides, with metamaterials, having polynomial law non-linearity, J. Modern Opt. 63 (2016), S32-S37.

[92] Yablonskii A.I., On rational solutions of the second Painlevé equation, Vesti AN BSSR, Ser. Fiz.-Tech. Nauk (1959), no. 3, 30-35. 